\title{
The connecting adolescents to reduce relapse (CARR) trial: study protocol for a randomized controlled trial comparing the efficacy of Groups 4 Health and cognitive behaviour therapy in young people
}

Tegan Cruwys ${ }^{1 *} \mathbb{D}$, Catherine Haslam², Zoe C. Walter ${ }^{2}$, Joanne Rathbone ${ }^{2}$ and Elyse Williams ${ }^{2}$

\begin{abstract}
Background: Depression is the leading cause of disability in young people (aged 15-25) globally. Loneliness is a major factor in the development and relapse of depression in young people, yet few interventions directly address loneliness. Groups 4 Health (G4H) - a novel, theoretically derived group psychotherapy intervention - may address this disconnect. Previous trials (Phase I and Phase II) have found G4H to be efficacious in reducing symptoms of depression. However, the efficacy of G4H compared to current evidence-based treatments (Phase III) has not been investigated. This protocol details the design and methodology of the Connecting Adolescents to Reduce Relapse (CARR) trial, a randomised control trial assessing the efficacy of G4H in young people relative to cognitive behavioural therapy (CBT).
\end{abstract}

Methods: The CARR trial is a two-arm non-inferiority randomised controlled trial that will compare the efficacy of $\mathrm{G} 4 \mathrm{H}$ to the most widely used evidence-based treatment for depression, CBT, at program completion and 6- and 12month follow up. Participants will be 200 young people (aged 15-25) with symptoms of depression and/or loneliness recruited from community and university mental health services. We hypothesise that the interventions will be comparable in reducing depression symptoms, but that $\mathrm{G} 4 \mathrm{H}$ will be superior in reducing loneliness. Because loneliness is a primary risk factor for depression relapse in young people, we therefore expect the benefits of Groups 4 Health to be particularly apparent at 12-month follow up.

Discussion: This trial will be the first to evaluate an intervention that targets loneliness, in comparison to the current gold standard treatment approach - CBT. If found to be effective, this program offers a new approach to treatment and relapse prevention of depression among young people.

Trial registration: Trial prospectively registered on ANZCTR (ACTRN12618000440224), registered on 27/03/2018.

Keywords: Social isolation, Loneliness, Group psychotherapy, Mood disorders, Depression, Mental health, Adolescence, Youth, Social identity

\footnotetext{
* Correspondence: tegan.cruwys@anu.edu.au

${ }^{1}$ Research School of Psychology, The Australian National University, Canberra,

ACT 2601, Australia

Full list of author information is available at the end of the article
}

(c) The Author(s). 2019 Open Access This article is distributed under the terms of the Creative Commons Attribution 4.0 International License (http://creativecommons.org/licenses/by/4.0/), which permits unrestricted use, distribution, and reproduction in any medium, provided you give appropriate credit to the original author(s) and the source, provide a link to the Creative Commons license, and indicate if changes were made. The Creative Commons Public Domain Dedication waiver (http://creativecommons.org/publicdomain/zero/1.0/) applies to the data made available in this article, unless otherwise stated. 


\section{Background}

Depression has been ranked as the single greatest cause of disability in young people aged 15-25 [1]. Although both pharmacological and psychotherapy treatments are effective in reducing acute symptoms in young people [2], they come with a range of downsides. The cost of these treatments can be prohibitive [3], particularly because depression is more prevalent among disadvantaged communities [4]. There is also a global shortfall of mental health professionals who have the level of training required to provide these evidence-based treatments. This shortfall is most apparent in areas of greatest need, such as in non-urban areas with high rates of socioeconomic deprivation [5].

There are other barriers to the effective treatment of depression, particularly for young people. Previous research suggests that only a minority of young people with depression speak to a health professional about it [6], and only a minority of those who do receive bestpractice treatment [2]. For instance, a common pathway to treatment is antidepressant medication prescribed following a consultation with a General Practitioner. However, the most common antidepressants prescribed for adults are not suitable for young people due to elevated suicide risk [7]. Even for those medications that are safe for adolescents, compliance rates are as low as 45\% [8], often attributed to side effects such as weight gain and drowsiness. While most young people prefer non-drug treatment [9], the stigma associated with therapy often leads to treatment avoidance [10]. For these reasons, there is a need to develop treatment alternatives for young people with depression that are cost-effective, non-stigmatising, and widely accessible.

Furthermore, although short-term treatments are generally effective at alleviating acute symptoms, existing approaches to relapse prevention are only minimally effective. About $80 \%$ of individuals who have experienced a depressive episode relapse, experiencing an average of four episodes across a lifetime [11]. Even among those who receive best practice treatment, approximately onethird relapse within 18 months [12]. Therefore, new innovations in treatment should prioritise reducing depression recurrence.

\section{Loneliness as a promising target for intervention}

Research has demonstrated that loneliness both precipitates and maintains depression. Longitudinal studies have shown that loneliness is a powerful predictor of depression onset, even after controlling for other candidate variables [13]. Loneliness also predicts poorer outcomes in depression treatment [14] and increases the risk of relapse [15]. These findings suggest that targeting loneliness may offer an effective approach to preventing relapse. Research has shown that when a person with depression joins a group, they reduce their risk of relapse four years later by approximately $24 \%$ [16].

Several diverse approaches to targeting loneliness have been developed [17]. These approaches mostly fall into two broad categories: either (1) befriending interventions, which seek to increase opportunities for social contact or provide social support, or (2) cognitive interventions which seek to address maladaptive cognitions or inadequate social skills. However, most of these interventions are not informed by theory, and evidence of their effectiveness is mixed [18, 19]. Indeed, Masi and colleagues stated that "loneliness interventions to date have not attained the degree of efficacy achieved by interventions targeting other social and behavioural outcomes" ([19], p.257). Furthermore, loneliness interventions have, by and large, been developed and evaluated for older adults, with little attention paid to young people. Given the evidence that adolescents and young adults report some of the highest levels of loneliness in the population [20], there is particular need for research in this area.

\section{Groups 4 health}

Groups 4 Health $(\mathrm{G} 4 \mathrm{H})$ was developed to fill this void as a theory-driven, group psychotherapy intervention that targets loneliness $[21,22]$. G4H is informed by the Social Identity Approach to Health (see [23, 24] for a summary), which is a conceptual framework that articulates how social group memberships, and the social identities associated with them, affect health and wellbeing.

To date, two trials of G4H have been completed. The first was a Phase I pilot [25], in which the program was delivered to 81 people $\left(M_{\text {age }}=20.95 ; S D=5.05\right)$ presenting with loneliness and affective disturbance (symptoms of depression or anxiety), of whom 54 completed $\mathrm{G} 4 \mathrm{H}$, and 26 completed the 6-month follow up. This group was compared to a matched, but not randomised, assessment-only control group. G4H was found to significantly improve mental health (depression, general anxiety, social anxiety, and stress), well-being (selfesteem, life satisfaction) and social connectedness (loneliness, social functioning), both on program completion and at 6-month follow up. Moreover, G4H recipients had significantly better outcomes than those in the control group at the 6-month follow up on measures of depression, anxiety, stress and self-esteem. In line with the theorised mechanisms of the G4H intervention, analyses also showed that participants whose identification with their G4H group and their subjective sense of belonging to multiple groups increased were most likely to experience improvements in depression, anxiety, stress, loneliness, and life satisfaction.

The second trial was a Phase II randomised control trial (RCT; [23]) in a sample of adults recruited from the 
community $\left(M_{\text {age }}=31.06 ; S D=12.80\right)$. This RCT examined the efficacy of G4H compared to a Treatment-AsUsual (TAU) control among people with current depression symptoms or health-professional diagnosis of a mental illness $(N=120)$. In this trial, the TAU group received a diverse range of interventions, with more than $50 \%$ receiving antidepressant medication, psychotherapy, or both. Intention-to-treat analyses showed that people in the G4H condition experienced a significant decline in loneliness, social anxiety and primary care attendance, and a significant increase in their sense of belonging to multiple groups, relative to TAU. There was also evidence of significant pre-post improvement in depression among those who completed $\mathrm{G} 4 \mathrm{H}$, although this was not significantly greater than the improvement seen in the TAU condition.

These data suggest that $\mathrm{G} 4 \mathrm{H}$ has good potential to benefit the mental health of young people in treatment. The next important stage is to test $\mathrm{G} 4 \mathrm{H}$ against a uniform, active treatment. This is the purpose of the present program of research with young adults with depression.

\section{Aims}

The project aims to evaluate $\mathrm{G} 4 \mathrm{H}$ in comparison to the current gold standard evidence-based psychological treatment for youth depression, CBT. Based on the existing evidence, we predict:

Hypothesis 1: At program completion, G4H will be superior to CBT in reducing loneliness (a major risk factor for relapse in depression).

Hypothesis 2: At program completion, $\mathrm{G} 4 \mathrm{H}$ will be as effective as CBT in reducing symptoms of depression (i.e., be non-inferior)

Hypothesis 3: The benefits of G4H for loneliness and depression will be sustained at 12-month follow up.

\section{Method}

\section{Trial design}

CARR is a two-arm non-inferiority parallel (1:1 ratio) randomised controlled trial. A summary of this study's compliance with Standard Protocol Items: Recommendations for Interventional Trials (SPIRIT), is provided in the Additional file 1.

\section{Participants}

Participants will be 200 young people presenting with psychological distress recruited from two sources in South East Queensland, Australia: (1) a community youth mental health service (headspace, five sites), and (2) a university psychology clinic (University of Queensland). The following criteria will be used in recruitment:

\section{Inclusion}

- Aged between 15 to 25 years

- English speaking

- The presence of depressive symptoms on the PHQ-9 ( $>=5$; corresponding to at least the mildly impairing clinical range; [23]); OR

- a health-professional diagnosed mental health condition (e.g., major depressive disorder); OR

- The presence of elevated loneliness on the UCLA (> = 40; approximately $1 \mathrm{SD}$ above the mean for adolescents; [26]).

\section{Exclusion}

- Currently in receipt of other evidence-based treatment for depression (psychotherapy or psychopharmacology)

- Current high risk suicidal ideation or severe selfharming behaviour.

- Current psychotic episode or severe personality disorder that would interfere with ability to participate in group work.

- Severe neurological condition or severe learning impairment that would hinder engagement with the content of the proposed interventions

- Current alcohol or other substance dependence / intoxication

Participants will be monitored throughout the trial for any change in these inclusion and exclusion criteria (e.g., in adjunct treatment status, or in suicide risk). Participants whose mental health declines for any reason will be provided with additional one-on-one supportive counselling by their group facilitator and referred to additional treatment where indicated. If a person who is initially eligible for the trial subsequently meets an exclusion criterion at any point during the activetreatment phase of the trial (T0-T5) they will be excluded from per-protocol analyses, but not intention-totreat analyses.

\section{Recruitment}

Allied health professionals and professionals-in-training at the two services will be invited to participate in an information session, which will include selection criteria for the participants, and a flyer for them to display within the service to inform participants of the program. These professionals will identify eligible patients for inclusion into the study on a rolling basis. They will approach potential participants and provide them with contact details for trial staff, including a website with 
links to the Participant Information Sheet and Consent Form. Research staff will contact potential participants to conduct screening and assessment, explain the study in more detail, answer any questions arising, and go through the consent process. If a potential participant indicates thoughts of self-harm in the baseline measures (i.e., a score of 3 or 4 on item 9 of the PHQ-9), a risk assessment will be conducted at this stage. Participants will provide fully informed, written and voluntary consent. Additionally, for those participants aged 16 or under, their parent or guardian will be informed of their participation and provided with an information sheet with the permission of the young person. However, primacy will be given to the consent of the young person, in line with the organisational policies of the research setting (which does not require parental consent for young people to access mental health services). Participants will be eligible to complete the CARR trial either as an (evidence-based) alternative to standard care, or while they are on a waiting list for standard care.

\section{Incentives}

Participants will not be offered incentives to undertake the intervention, other than receipt of free, evidencebased mental health care. Those participants who are also students enrolled in first-year psychology courses will receive course credit for their participation in the program. To improve attendance at Module 5, where post-program assessments are conducted, all participants will be offered either a coffee or supermarket voucher (AUD\$15 value) for attendance. To minimise attrition, participants will be offered AUD\$50 incentive to complete each of the 6-month and 12-month follow up assessments. Participants will be followed up unless they actively withdraw from the trial (i.e., data will be sought from participants who did not attend group sessions or who no longer meet inclusion criteria, in order to honour the intention-to-treat principle).

\section{Randomisation}

Participants who are eligible will be randomly assigned by the research team following simple randomisation procedures (computerized random numbers using Excel random number generator feature) to either $\mathrm{G} 4 \mathrm{H}$ or CBT using a 1:1 ratio. Participants will be randomised at the group level once sufficient participants have been recruited for a group (minimum of 5). That is, participants will first be recruited into a treatment group, and each group will then be randomised to receive either $\mathrm{G} 4 \mathrm{H}$ or CBT, using the randomisation process outlined above. Neither the participant nor the research team will be blind to intervention status. Assessment of primary outcomes at each timepoint will occur via a web interface to ensure that this is not affected by intervention status.

\section{Intervention}

Participants will be randomly assigned to receive either G4H or CBT. Groups will not commence unless a minimum of 5 people have consented to participate, with the maximum number of people recruited to each group being 9 people. Interventions are matched for contact time and mode of delivery, and both include five group sessions of 60-90 min in duration, run weekly for the first four weeks, and a final "booster" session that is held one month later. Postgraduate students in professional psychology (with provisional registration) will facilitate the groups ( 2 facilitators per group). Treatment fidelity and consistency of program delivery will be maintained by providing: a half-day of training, weekly group supervision from a registered clinical psychologist, direct observation by investigators to ensure quality, and regular team meetings with the research team. Additionally, G4H facilitators will anonymously complete a brief questionnaire at the end of each session to assess (a) what session activities were covered, (b) the length of session, and (c) facilitator session satisfaction and ease of delivery. Facilitators will also record participant attendance.

\section{Groups 4 health (G4H)}

$\mathrm{G} 4 \mathrm{H}$ is a manualised, five-session group-based intervention designed to reduce loneliness through facilitating social group integration $[21,22]$. The program is comprised of five modules - the contents of which are summarised in Fig. 1 below.

Module 1: Schooling This module seeks to raise awareness of the beneficial effects that social group memberships have for health. Module 1 highlights the costs of ignoring the social dimensions of health and points out that using all of the social resources at our disposal generally leads to optimal health outcomes. The module also emphasises people's power to counter these effects by learning how best to develop, maintain and harness group-based resources.

Module 2: Scoping Explores each person's current group-based resources. This module engages participants in the process of social identity mapping [25]. This tool was developed to explore respondents' social identities in a way that is engaging, visual, and concrete. The map is then used to facilitate a discussion of how people would ideally like their groups to look in the future and identify any gaps in group networks.

Module 3: Sourcing Targets development of strategies needed to identify and strengthen existing valued social identities. The goal of Module 3 is to assist people to optimize and sustain their group memberships over the 


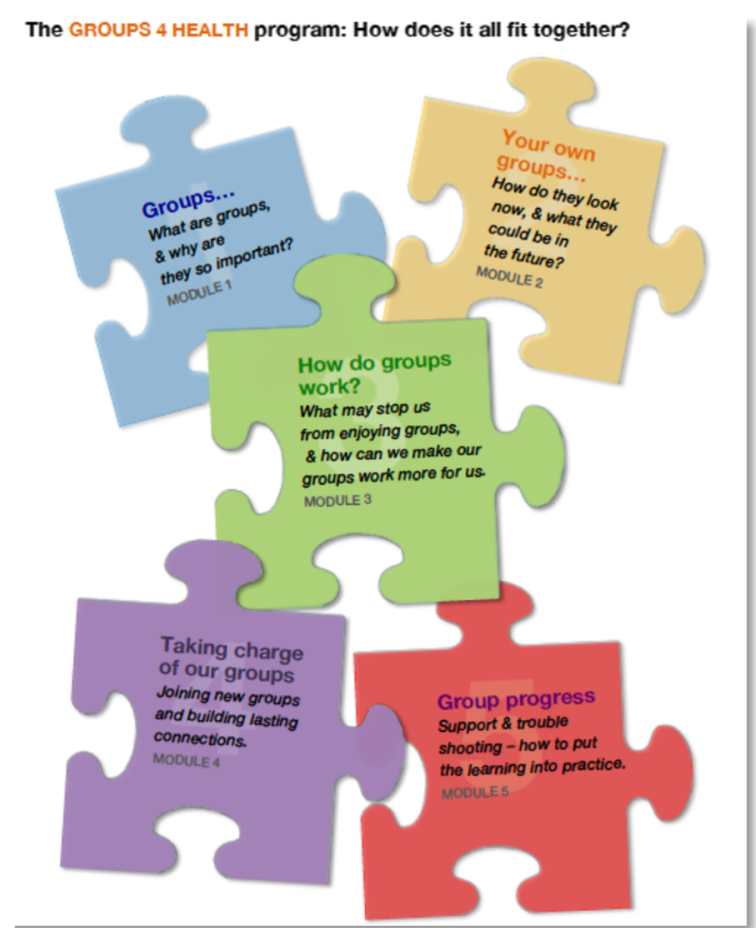

Schooling: Raising awareness of the value of groups for

health and of ways to harness this.

Scoping: Developing social maps to identify existing

connections and areas for social growth.

Sourcing: Training skills to maintain \& utilise existing

networks \& reconnect with valued groups

Scaffolding: Using group as a platform for new

social connections \& to train effective engagement.

Sustaining: Reinforcing key messages \& trouble-shooting

("booster" session one month later).

Fig. 1 Overview of the Groups 4 Health Modules

long term. Participants develop solutions to barriers to making the most of existing groups.

Module 4: Scaffolding Uses the G4H group as an exemplar for developing and embedding new social group connections, whilst also identifying which connections to develop. Group members are facilitated to prepare a social plan of action. These social plans are then trialled between this and the final module, which takes place one month later.

Module 5: Sustaining Is a booster session, held one month after Module 4 that seeks to troubleshoot any problems that people have encountered in implementing their social plans. Module 5 also revisits social identity maps, created in Module 2, to see how they have changed over the course of the program. The skills that have been learned across the previous modules are reviewed and key messages reinforced with the goal of encouraging long-term maintenance.

The program is supported by a facilitator manual and client workbook. The workbook contains a summary of the key learnings for each session, with sufficient space to complete activities (both within the sessions and as homework) and document any relevant notes and plans to achieve a person's individual goals.

\section{Group cognitive behaviour therapy}

The active comparison condition will involve the delivery of the current best-practice intervention for the treatment of adolescent depression: CBT. Specifically, an existing brief manualised program for group-based CBT for depression [27] will be used with minor adaptations to ensure comparable structure and amount of face-toface contact as the G4H condition. This specific manualised version of CBT has been found to be effective in reducing depression symptoms in a sample of high-risk adolescents relative to active control groups $(N=341$; [28]). Indeed, a meta-analysis found that this particular manualised program showed greater effect sizes than similar interventions with longer duration [29].

The manualised program involves the following components: (1) psychoeducation, in which participants learn about the link between thoughts, mood, and behaviour; (2) behavioural activation, in which participants learn strategies to increase activities that boost their sense of pleasure and achievement; and (3) cognitive restructuring, in which participants learn strategies for challenging and reframing unhelpful thinking patterns that maintain depression. The program also includes homework tasks and group-based activities.

\section{Measures}

Each participant will be asked to complete online surveys (or paper-and-pencil if they prefer) at eight time points. 
The full questionnaire package measuring all outcomes of interest will be administered before the intervention (T0), on its completion (T5), 6 months later (T6) and 12 months later (T7) - see Table 1 for a summary. These should take about 10-20 min on each occasion. In addition, a brief one-page questionnaire will be administered at the end of each session (T1, T2, T3, and T4), which will (only) include measures of depression symptoms, loneliness, module feedback, and group identification.

\section{Primary outcomes}

Depression, Anxiety and Stress Scale [30]. This measure comprises 21 items, 7 for each construct, for example "I felt that life was meaningless", rated on a 4-point scale $(0=$ did not apply to me at all, $3=$ applied to me very much, or most of the time. It has been validated for use with adolescents as young as 11 years in Australia [31], with an improvement greater than 3 points considered clinically significant [32]. The full DASS-21 (including anxiety and stress subscales) will be administered at T0, T5, T6 and T7, while the depression subscale will be administered at all timepoints T0-T7. The 9-item Patient Health Questionnaire is included as an additional measure of depression (T0, T5, T6, T7); this will be used as a screening tool rather than as the primary outcome measure.

UCLA-20: The UCLA Loneliness scale has been validated for use with a young adult sample [33, 34]. It comprises 20 items, for example "Felt isolated from others" requiring a response on a 4 -point scale $(1=$ never, $4=$ often). The full scale will be included at T0, T5, T6 and $\mathrm{T} 7$, and a 4-item version of the UCLA scale will be included in the end of session questionnaire (T1-T4).

\section{Secondary outcomes Social anxiety}

This was assessed with the Social Phobia Inventory (SPIN; [35]), comprising 17 items (e.g., "Being embarrassed or looking stupid are among my worst fears"). Each item is rated on a 5 -point scale $(1=$ not at all, $5=$

Table 1 Overview of CARR measures and timepoints of measurement

\begin{tabular}{|c|c|c|c|c|c|c|c|c|c|}
\hline Construct & Measure & $\begin{array}{l}\text { T0 } \\
\text { Baseline }\end{array}$ & $\begin{array}{l}\text { T1 } \\
\text { After } \\
\text { session } 1\end{array}$ & $\begin{array}{l}\mathrm{T} 2 \\
\text { After } \\
\text { session } 2\end{array}$ & $\begin{array}{l}\text { T3 } \\
\text { After } \\
\text { session } 3\end{array}$ & $\begin{array}{l}\text { T4 } \\
\text { After } \\
\text { session } 4\end{array}$ & $\begin{array}{l}\text { T5 } \\
\text { Post- } \\
\text { treatment }\end{array}$ & $\begin{array}{l}\text { T6 } \\
\text { 6-month } \\
\text { follow up }\end{array}$ & $\begin{array}{l}\text { T7 } \\
\text { 12-month } \\
\text { follow up }\end{array}$ \\
\hline \multirow[t]{4}{*}{ Primary outcomes } & Depression (DASS-21) & $\cdot$ & $\cdot$ & $\cdot$ & $\cdot$ & $\cdot$ & $\cdot$ & $\cdot$ & $\cdot$ \\
\hline & Depression (PHQ-9) & $\cdot$ & & & & & $\cdot$ & $\cdot$ & $\cdot$ \\
\hline & Loneliness (4 item) & $\cdot$ & $\cdot$ & $\cdot$ & $\cdot$ & $\cdot$ & $\cdot$ & $\cdot$ & $\cdot$ \\
\hline & Loneliness (20 item) & $\cdot$ & & & & & $\cdot$ & $\cdot$ & $\cdot$ \\
\hline \multirow[t]{6}{*}{ Secondary outcomes } & Social anxiety & $\cdot$ & & & & & $\cdot$ & $\cdot$ & $\cdot$ \\
\hline & GP attendance (last month) & $\cdot$ & & & & & $\cdot$ & $\cdot$ & • \\
\hline & Wellbeing & $\cdot$ & & & & & $\cdot$ & $\cdot$ & · \\
\hline & Life satisfaction & $\cdot$ & & & & & $\cdot$ & $\cdot$ & $\cdot$ \\
\hline & Self esteem & · & & & & & $\cdot$ & $\cdot$ & $\cdot$ \\
\hline & Subjective physical health & $\cdot$ & & & & & $\cdot$ & $\cdot$ & • \\
\hline \multirow[t]{6}{*}{ Process measures } & Multiple group membership & $\cdot$ & & & & & $\cdot$ & $\cdot$ & • \\
\hline & Multiple group compatibility & $\cdot$ & & & & & $\cdot$ & $\cdot$ & $\cdot$ \\
\hline & Mental illness identity & $\cdot$ & & & & & $\cdot$ & $\cdot$ & · \\
\hline & Group cohesion & & & & & & . & & \\
\hline & Therapy group identification & & $\cdot$ & $\cdot$ & $\cdot$ & $\cdot$ & $\cdot$ & & \\
\hline & Service identification & & & & & & $\cdot$ & & \\
\hline \multirow[t]{7}{*}{ Acceptability/feasibility } & Useful & & $\cdot$ & $\cdot$ & $\cdot$ & $\cdot$ & $\cdot$ & $\cdot$ & $\cdot$ \\
\hline & Enjoyed & & & & & & $\cdot$ & $\cdot$ & $\cdot$ \\
\hline & Interesting & & & & & & $\cdot$ & $\cdot$ & $\cdot$ \\
\hline & Learnt something & & & & & & $\cdot$ & $\cdot$ & · \\
\hline & Homework completion & & $\cdot$ & $\cdot$ & $\cdot$ & $\cdot$ & $\cdot$ & & \\
\hline & Facilitator questionnaire & & $\cdot$ & $\cdot$ & · & $\cdot$ & $\cdot$ & & \\
\hline & Attendance & & $\cdot$ & $\cdot$ & $\cdot$ & $\cdot$ & $\cdot$ & & \\
\hline
\end{tabular}

Notes: DASS21 Depression Anxiety Stress Scales (21 item) PHQ-9 Patient Health Questionnaire (9 item).

GP General Practitioner 
extremely), with higher scores indicating greater social anxiety.

\section{General practitioner (GP) visits}

This was measured with a single item: "How many times have you been to see a general practitioner (medical doctor) in the last month" (as used in $[25,36]$ ).

\section{Subjective wellbeing}

The Short Warwick Edinburgh Mental Wellbeing Scale [37] will be used to index well-being. It is a 7 item scale with positively worded items (e.g., "I've been thinking clearly"), which respondents rate for how often they have experienced them over the past two weeks, on a 5 -point scale $(1=$ none of the time; to $5=$ all of the time).

\section{Life satisfaction}

The Satisfaction With Life Scale (SWLS; [38]) will be included to assess global life satisfaction. Participants will respond to 5 items (e.g., "I am satisfied with my life") on a 7 -point scale ( 1 = strongly disagree, 7 -strongly agree).

\section{Self esteem}

This was measured using the Single Item Self Esteem measure (SISE; [39]). Participants responded to the item "I have high self esteem" on a 4-point scale $(1=$ not at all true of me, $4=$ very true of me).

\section{Subjective physical health}

Participant's self-rated health status was measured using a single item "My current overall health is...", with responses ranging from 1 (very poor) to 7 (very good). This measure is widely used in community and population health studies and has been found to be a valid predictor of physical health and mortality risk [40].

\section{Process measures}

Five measures will be used to index different aspects of social identification and group processes as follows:

(i). Social identification with treatment group: This is a 4-item scale [41] was used to assess identification with the treatment group (e.g., "I identify with my G4H group/CBT group") and is rated on a 7-point scale ( $1=$ strongly disagree, $7=$ strongly agree).

(ii). Social identification with service: This is a 4-item scale [41] was used to assess identification with the service (e.g., "I identify with Headspace/the UQ Psychology Clinic") and is rated on the same 7 point scale used above ( $1=$ strongly disagree, 7 = strongly agree).

(iii) Multiple group membership. This will be measured using the Multiple Group Membership scale from the Exeter Identity and Transition Scales [42]. The 3-item version of the scale was used to index people's strength of connectedness to multiple groups [43]. Participants are asked to rate each item (e.g., "I am a member of lots of different social groups") on a 5 -point scale $(1=$ do not agree at all, $5=$ agree completely).

(iv) Social identity centrality: This is a 7-item scale adapted from [44] assessing the psychological salience of a particular social category membership, here as someone with mental health problems (e.g., "I often think about the fact that I am a person who has problems with mental health"; 1 = do not agree at all, $5=$ agree completely).

(v). Group Cohesion. The Group Environment Scale Cohesion subscale [45] will be used to measure treatment group cohesion. Nine items (e.g., "There is a strong feeling of belongingness in this group") are measured on a 7 -point scale $(1=$ not at all, $7=$ very much).

\section{Acceptability and feasibility}

Participants will be asked to rate their perceived usefulness of the program after every session (T1-T5): "How useful did you find today's module?" on a scale from 1 (not useful at all) to 10 (very useful). At T5, T6, and T7, participants will additionally be asked to rate how much they enjoyed the program, found the program interesting, learnt from the program, and how likely they were to use the techniques they learned during the program (each on a 1-10 scale). Facilitators will also provide a rating of the perceived usefulness, relevance, learning, and enjoyment for participants after each module (T1T5).

Qualitative feedback on the program will be sought from participants and facilitators, both in the form of written feedback (at T1-T7) as well as in a series of focus groups conducted separately with a subgroup of participants (including those who withdrew from the program) and facilitators.

\section{Sample size power calculations}

The CARR sample size was determined by power calculations for a non-inferiority trial, using effect sizes and retention rates from our published pilot study. We have estimated the non-inferiority margin based on historical evidence for the effect size of the active comparator (based on recommendations by [46] and [47]). The effect size of this CBT program versus control was $d=0.49$ at 6 month follow up (recalculated from [28] using methods recommended by [19]). This corresponds to 2.2 points on the DASS-21 depression subscale (using the expected standard deviation for an adolescent population of 4.53; [31]). To have 0.80 power to detect this difference with an alpha level of 0.05, 67 people per 
condition would need to be retained at follow up. Assuming 50\% eligibility and $67 \%$ survival rates, which are consistent with our pilot data, we anticipate that screening 400 people for eligibility (see Fig. 2) will lead to retention of $N=67$ per condition at follow up [48]. However, this is a conservative estimate of retention due to the addition of incentives for follow up data completion in this trial [49].

\section{Analyses}

Data quality will be improved by using a web interface that prompts (but does not force) participants to complete missing items. This will reduce missing data and prevent errors from manual data entry. Data quality will be checked by assessing ranges for each variable and interrogating outliers. We will examine the differences in baseline characteristics between the groups descriptively. Since differences between randomised groups at baseline could have occurred by chance, no formal significance testing will be conducted (in accordance with recommendations by [50]). No covariates will be included in the primary analysis (in accordance with recommendations by [51]). However, we will include exploratory analyses which assess the interaction between condition and baseline characteristics where indicated.

To assess the effect of intervention, group differences on the primary and secondary outcome measures, with all available timepoints included in the model, will be examined using a series of mixed effects repeated measures (MMRM) models, which will specify timepoint, participant, and therapy group as levels in the analyses. MMRM is a full information maximisation likelihood estimation strategy that can model data even when some observations are missing, and thus honours the intention-to-treat principle [52]. For all hypothesis testing, both intention-to-treat analyses (ITT; where all available data are included in MMRM) and per protocol analyses (PP; only including participants who met eligibility criteria throughout the trial period, were randomly assigned, completed the intervention according to the protocol, and had both baseline and follow up data) will be completed. Best fit, as determined by Information

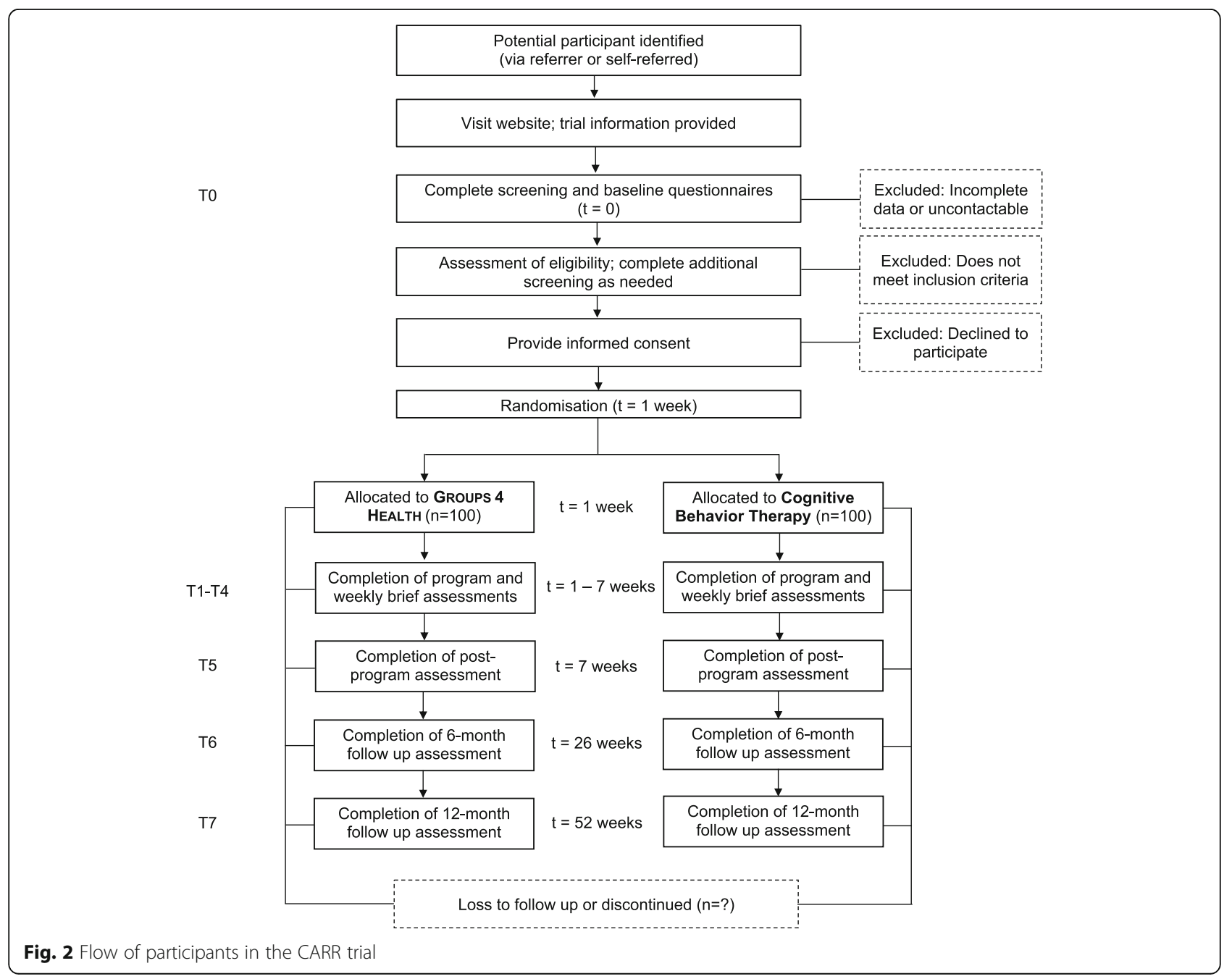


Criteria (e.g. AIC; BIC) method will be used for determining the covariance structure for MMRM models.

$\mathrm{H} 1$ will be considered supported if there is evidence of significant improvements in loneliness in the G4H condition (H1a), and a significant condition by time interaction, such that improvements in loneliness over time will be greater for the $\mathrm{G} 4 \mathrm{H}$ condition than in the CBT condition (H1b). H2 will be considered supported if there is evidence of significant improvements in depression in both conditions, with a non-significant condition by time interaction. The non-inferiority margin will be a DASS-21 depression change score difference of 2.20. H3 will be considered supported if there is no significant increase in depression and loneliness scores between T6 and $\mathrm{T} 7 \mathrm{in}$ the G4H condition.

\section{Discussion}

Depression is a leading cause of disability among young people. Although there are currently evidence-based pharmacological and non-pharmacological treatments for depression, young people face barriers to treatment, including side effects, cost, accessibility, and stigma. Additionally, relapse after treatment is common [12].

Given this, it is important to develop interventions that are accessible to young people and target primary causes of depression and relapse. Loneliness is a primary cause of depression onset and relapse in young people [53]. The current protocol outlines a non-inferiority RCT to assess the efficacy of a novel intervention that targets loneliness in young people, compared to the current gold standard evidence-based psychological treatment for depression (CBT). If found to be effective, this program offers a new approach to treatment and relapse prevention of depression among young people that is cost-effective, accessible, and destigmatising.

\section{Additional file}

Additional file 1: SPIRIT 2013 Checklist: Recommended items to address in a clinical trial protocol and related documents*. (PDF 85 kb)

\author{
Abbreviations \\ AIC: Akaike Information Criterion; ANZCTR: Australian New Zealand Clinical \\ Trials Registry; BIC: Bayes Information Criterion; CARR: Connecting \\ Adolescents to Reduce Relapse; CBT: Cognitive Behaviour Therapy; \\ DASS21: Depression, Anxiety, Stress Scales - 21 item; G4H: Groups 4 Health; \\ ITT: Intention to treat; MMRM: Mixed model repeated measures; \\ NHMRC: National Health and Medical Research Council; PHQ-9: Patient \\ Health Questionnaire - 9 item depression scale; PP: Per protocol; \\ RCT: Randomised Control Trial
}

\section{Acknowledgements}

We acknowledge Victoria Gore-Jones and Frances Dark for their support in the early stage of protocol development, grant application, and the ethical review process. We acknowledge Genevieve Dingle, Alex Haslam, Jolanda Jetten, and Sarah Bentley for their co-authorship of G4H. We acknowledge the graduate psychology students who facilitated the program and all the young people involved in the trial.

\section{Authors' contributions}

TC designed the trial, wrote the protocol, and led the project. $\mathrm{CH}$ led the development of $\mathrm{G} 4 \mathrm{H}$ and provided critical input at all stages of the project. TC and $\mathrm{CH}$ trained group facilitators and TC supervised all facilitators. ZW provided guidance on the adaptation of the protocol for publication. JR and EW are the trial managers for the project. All authors provided feedback on the manuscript. All authors read and approved the final manuscript.

\section{Funding}

Funding was provided by Rotary Health Australia to $\mathrm{TC}$ and $\mathrm{CH}$. The funder had no role in the design of research, analyses, or decision to publish.

\section{Availability of data and materials}

Data sharing is not applicable to this article as no datasets were generated or analysed in the preparation of the trial protocol.

\section{Ethics approval and consent to participate}

Ethical approval for this trial, including the consent procedure, was provided by the Brisbane Metro South Human Research Ethics Committee (HREC/18/ QPAH/54) and the University of Queensland (\#2018000420). Informed and voluntary consent was obtained in writing from all participants. Parents of young people aged 15 or 16 were informed of their participation with the young person's consent, in accordance with the organisational policy of the healthcare partner site. The authors assert that all procedures contributing to this work comply with the ethical standards of the relevant national and institutional committees on human experimentation and with the Helsinki Declaration of 1975, as revised in 2008

\section{Consent for publication}

Not applicable.

\section{Competing interests}

$\mathrm{TC}$ and $\mathrm{CH}$ are authors of the $\mathrm{G} 4 \mathrm{H}$ program. As training in this program is run at cost and includes provision of the resources, they have an intellectual, rather than financial, interest in the program.

\section{Author details}

${ }^{1}$ Research School of Psychology, The Australian National University, Canberra, ACT 2601, Australia. ${ }^{2}$ School of Psychology, University of Queensland, Brisbane 4072, QLD, Australia.

Received: 7 May 2019 Accepted: 20 May 2019

Published online: 20 June 2019

\section{References}

1. Vos T, Barber RM, Bell B, Bertozzi-Villa A, Biryukov S, Bolliger l, et al. Global, regional, and national incidence, prevalence, and years lived with disability for 301 acute and chronic diseases and injuries in 188 countries, 1990-2013: a systematic analysis for the global burden of disease study 2013. Lancet. 2015;386(9995):743-800

2. APA. Practice guideline for the treatment of patients with Major Depressive Disorder, 3rd ed.; 2010. Available at: http://psychiatryonline.org/pdfaccess. ashx?ResourcelD=243261\&PDFSource $=6$.

3. Wang PS, Simon G, Kessler RC. The economic burden of depression and the cost-effectiveness of treatment. Int J Methods Psychiatr Res. 2003;12(1):22-33.

4. Simon GE, Fleck M, Lucas R, Bushnell DM. Prevalence and predictors of depression treatment in an international primary care study. Am J Psychiatry. 2004:161(9):1626-34.

5. Saxena S, Thornicroft G, Knapp M, Whiteford $H$. Resources for mental health: scarcity, inequity, and inefficiency. Lancet. 2007;370:878-89.

6. Mojtabai R, Olfson M, Han B. National trends in the prevalence and treatment of depression in adolescents and young adults. Pediatrics. 2016; 138(6):e20161878

7. Ma D, Zhang Z, Zhang X, Li L. Comparative efficacy, acceptability, and safety of medicinal, cognitive-behavioral therapy, and placebo treatments for acute major depressive disorder in children and adolescents: a multipletreatments meta-analysis. Curr Med Res Opin. 2014;30(6):971-95.

8. Sawada N, Uchida H, Suzuki T, Watanabe K, Kikuchi T, Handa T, Kashima H. Persistence and compliance to antidepressant treatment in patients with depression: a chart review. BMC Psychiatry. 2009;9:38. 
9. Rokke PD, Scogin F. Depression treatment preferences in younger and older adults. J Clin Geropsychol. 1995;1(3):243-57.

10. Crabtree JW, Haslam SA, Postmes T, Haslam C. Mental health support groups, stigma, and self-esteem: positive and negative implications of group identification. J Soc Issues. 2010;66(3):553-69.

11. Judd LL. The clinical course of unipolar major depressive disorders. Arch Gen Psychiatry. 1997;54(11):989-91.

12. Shea MT, Elkin I, Imber SD, Sotsky SM, Watkins JT, Collins JF, et al. Course of depressive symptoms over follow-up: findings from the National Institute of Mental Health treatment of depression collaborative research program. Arch Gen Psychiatry. 1992;49(10):782-7.

13. Cacioppo JT, Hawkley LC, Thisted RA. Perceived social isolation makes me sad: 5-year cross-lagged analyses of loneliness and depressive symptomatology in the Chicago health, aging, and social relations study. Psychol Aging. 2010;25(2):453.

14. Trivedi MH, Morris DW, Pan J, Grannemann BD, John Rush A, Rush AJ. What moderator characteristics are associated with better prognosis for depression? Neuropsychiatr Dis Treat. 2005;1(1):51-7.

15. Backs-Dermott BJ, Dobson KS, Jones SL. An evaluation of an integrated model of relapse in depression. J Affect Disord. 2010;124(1-2):60-7.

16. Cruwys T, Dingle GA, Haslam SA, Haslam C, Jetten J, Morton TA. Social group memberships protect against future depression, alleviate depression symptoms and prevent depression relapse. Soc Sci Med. 2013;98:179-86.

17. Cacioppo S, Grippo AJ, London S, Goossens L, Cacioppo JT. Loneliness: clinical import and interventions. Perspect Psychol Sci. 2015;10(2):238-49.

18. Mann F, Bone JK, Lloyd-Evans B, Frerichs J, Pinfold V, Ma R, Wang J, Johnson $\mathrm{S}$. A life less lonely: the state of the art in interventions to reduce loneliness in people with mental health problems. Soc Psychiatry Psychiatr Epidemiol. 2017:52(6):627-38.

19. Masi CM, Chen HY, Hawkley LC, Cacioppo JT. A meta-analysis of interventions to reduce loneliness. Personal Soc Psychol Rev. 2011;15(3):219-66.

20. Mance, P. (2018). Is Australia experiencing an epidemic of loneliness? Evidence from 16 waves of the Housing, Income and Labour Dynamics in Australia Survey. Working Paper, 2018. Canberra: Relationships Australia. https://www.relationships. org.au/what-we-do/research/an-epidemic-of-loneliness-2001-2017.

21. Haslam C, Cruwys T, Haslam SA, Bentley SV, Dingle GA, Jetten J. GRoups 4 HEALTH manual (version 3.0). Social identity and groups network (SIGN): University of Queensland, Australia; 2016a.

22. Haslam C, Cruwys T, Haslam SA, Bentley SV, Dingle GA, Jetten J. Groups 4 HEALTH workbook. (version 3.0). Social identity and groups network (SIGN): University of Queensland, Australia; 2016b.

23. Haslam C, Jetten J, Cruwys T, Dingle GA, Haslam SA. The new psychology of health: unlocking the social cure. London and New York: Routledge; 2019.

24. Jetten J, Haslam, SA, Cruwys T, Greenaway KH., Haslam C, Steffens NK. Advancing the social identity approach to health and well-being: progressing the social cure research agenda. Eur J Soc Psychol. 2017;47: 789-802. https://doi.org/10.1002/ejsp.2333.

25. Haslam C, Cruwys T, Haslam SA, Dingle G, Chang MX. GROUPS 4 HEALTH: evidence that a social-identity intervention that builds and strengthens social group membership improves mental health. J Affect Disord. 2016;194:188-95.

26. Shevlin M, Murphy S, Murphy J. The latent structure of loneliness: testing competing factor models of the UCLA loneliness scale in a large adolescent sample. Assessment. 2015;22:208-15.

27. Stice E, Burton E, Bearman SK, Rohde P. Randomized trial of a brief depression prevention program. Behav Res Ther. 2007:45(5):863-76.

28. Stice E, Rohde P, Seeley JR, Gau JM. Brief cognitive-behavioral depression prevention program for high-risk adolescents outperforms two alternative interventions: a randomized efficacy trial. J Consult Clin Psychol. 2008;76(4): 595-606.

29. Stice E, Shaw H, Bohon C, Marti CN, Rohde P. A meta-analytic review of depression prevention programs for children and adolescents: factors that predict magnitude of intervention effects. J Consult Clin Psychol. 2009;77(3): 486-503.

30. Lovibond SH, Lovibond PF. Manual for the depression anxiety stress scales. 2nd ed. Sydney: Psychology Foundation; 1995.

31. Szabo M, Szabo M. The short version of the depression anxiety stress scales (DASS-21): factor structure in a young adolescent sample. J Adolesc. 2015; 33(1):1-8.

32. Page AC, Hooke GR, Morrison DL. Psychometric properties of the depression anxiety stress scales (DASS) in depressed clinical samples. Br J Clin Psychol. 2007:46:283-97.
33. Mahon NE, Yarcheski TJ, Yarcheski A. Validation of the revised UCLA loneliness scale for adolescents. Research in Nursing \& Health. 1995;18(3): 263-70.

34. Russel D, Peplau LA, Cutrona CE. The revised UCLA loneliness scale: concurrent and discriminant validity evidence. J Pers Soc Psychol. 1980; 39(3):472-80.

35. Connor KM, Davidson JRT, Churchhill LE, Sherwood A, Foa E, Weisler RH. Psychometric properties of the social phobia inventory (SPIN): new selfrating scale. Br J Psychiatry. 2000;176:379-86.

36. Vedsted $P$, Christensen MB. Frequent attenders in general practice care: a literature review with special reference to methodological considerations. Public Health. 2005;119(2):118-37.

37. Stewart-Brown S, Tennant A, Tennant R, Platt S, Parkinson J, Weich S. Internal construct validity of the Warwick-Edinburgh mental well-being scale (WEMWBS): a Rasch analysis using data from the Scottish health education population survey. Health Qual Life Outcomes. 2009;7(1):15-22. https://doi.org/10.1186/1477-7525-7-15.

38. Diener E, Emmons RA, Larsen RJ, Griffin S. The satisfaction with life scale. J Pers Assess. 1985;49(1):71-5.

39. Robins RW, Hendin HM, Trzesniewski KH. Measuring global self-esteem: construct validation of a single-item measure and the Rosenberg selfesteem scale. Personal Soc Psychol Bull. 2001:27:151-61.

40. Idler EL, Benyamini Y. Self-rated health and mortality : a review of twentyseven community studies. J Health Soc Behav. 1997;38(1):21-37.

41. Postmes T, Haslam SA, Jans L. A single-item measure of social identification: reliability, validity and utility. Br J Soc Psychol. 2013:52:597-617.

42. Haslam C, Holme A, Haslam SA, lyer A, Jetten J, Williams WH. Maintaining group memberships: social identity continuity predicts well-being after stroke. Neuropsychological Rehabilitation. 2008;18:671-91.

43. Jetten J, Branscombe NR, Haslam SA, Haslam C, Cruwys T, Jones JM, Zhang, A. Having a lot of a good thing: multiple important group memberships as a source of self-esteem. Plos One. 2015;10(5):e0124609. https://doi.org/10. 1371/journal.pone.0124609.

44. Cameron JE. A three-factor model of social identity. Self Identity. 2004;3(3): 239-62.

45. Moos RH, Insel PM, Humphrey B. Preliminary manual for family environment scale, work environment scale, group environment scale: Consulting Psychologists Press; 1974.

46. Kaul S, Diamond GA. Good enough: a primer on the analysis and interpretation of noninferiority trials. Ann Intern Med. 2006a;145:62-9.

47. Althunian TA, de Boer A, Klungel OH, Insani WN, Groenwold RHH. Methods of defining the non-inferiority margin in randomized, double-blind controlled trials: a systematic review. Trials. 2017;18(1):1-9.

48. Julious SA. Sample sizes for clinical trials with Normal data. Statistical Medicine. 2004;23:1921-86

49. Cotter RB, Burke JD, Stouthamer-Loeber M, Loeber R. Contacting participants for follow-up: how much effort is required to retain participants in longitudinal studies? Evaluation and Program Planning. 2005;28(1):15-21 https://doi.org/10.1016/j.evalprogplan.2004.10.002.

50. Pocock SJ, McMurray JJV, Collier TJ. Statistical controversies in reporting of clinical trials: part 2 of a 4 part series on statistics of clinical trials. J Am Coll Cardiol. 2015;66(23):2648-62.

51. Kraemer HC. A source of false findings in published research studies: adjusting for covariates. JAMA Psychiatry. 2016;72(10):2015-6 https://doi.org/ 10.1001/jamapsychiatry.2015.1178.

52. Molenberghs G, Thijs H, Jansen I, Beunckens C, Kenward MG, Mallinckrodt C Carroll RJ. Analyzing incomplete longitudinal clinical trial data. Biostatistics. 2004;5(3):445-64 https://doi.org/10.1093/biostatistics/kxh001.

53. Hammen C. Adolescent depression: stressful interpersonal contexts and risk for recurrence. Curr Dir Psychol Sci. 2009;18(4):200-4.

\section{Publisher's Note}

Springer Nature remains neutral with regard to jurisdictional claims in published maps and institutional affiliations. 